\title{
Transcranial magnetic stimulation in neurology
}

\section{A review of established and prospective applications}

\author{
Mark C. Eldaief, MD \\ Daniel Z. Press, MD \\ Alvaro Pascual-Leone, MD, PhD
}

\section{Summary}

Transcranial magnetic stimulation (TMS) is a neurophysiologic technique to noninvasively induce a controlled current pulse in a prespecified cortical target. This can be used to transiently disrupt the function of the targeted cortical region and explore causal relations to behavior, assess cortical reactivity, and map out functionally relevant brain regions, for example during presurgical assessments. Particularly when applied repetitively, TMS can modify cortical excitability and the effects can propagate trans-synaptically to interconnected cortical, subcortical, and spinal cord regions. As such, TMS can be used to assess the functional integrity of neural circuits and to modulate brain activity with potential therapeutic intent.

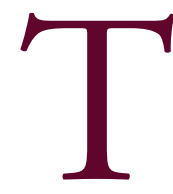

ranscranial magnetic stimulation (TMS) has emerged as a major tool used in the field of noninvasive brain stimulation. TMS operates on Faraday's principle of electromagnetic induction by which the transmission of a large, brief pulse of current through loops of copper wire (i.e., magnetic coil) give rise to a fluctuating magnetic field perpendicular to the plane of the coil that subsequently induces an electric field predominantly parallel to the inner surface of the volume

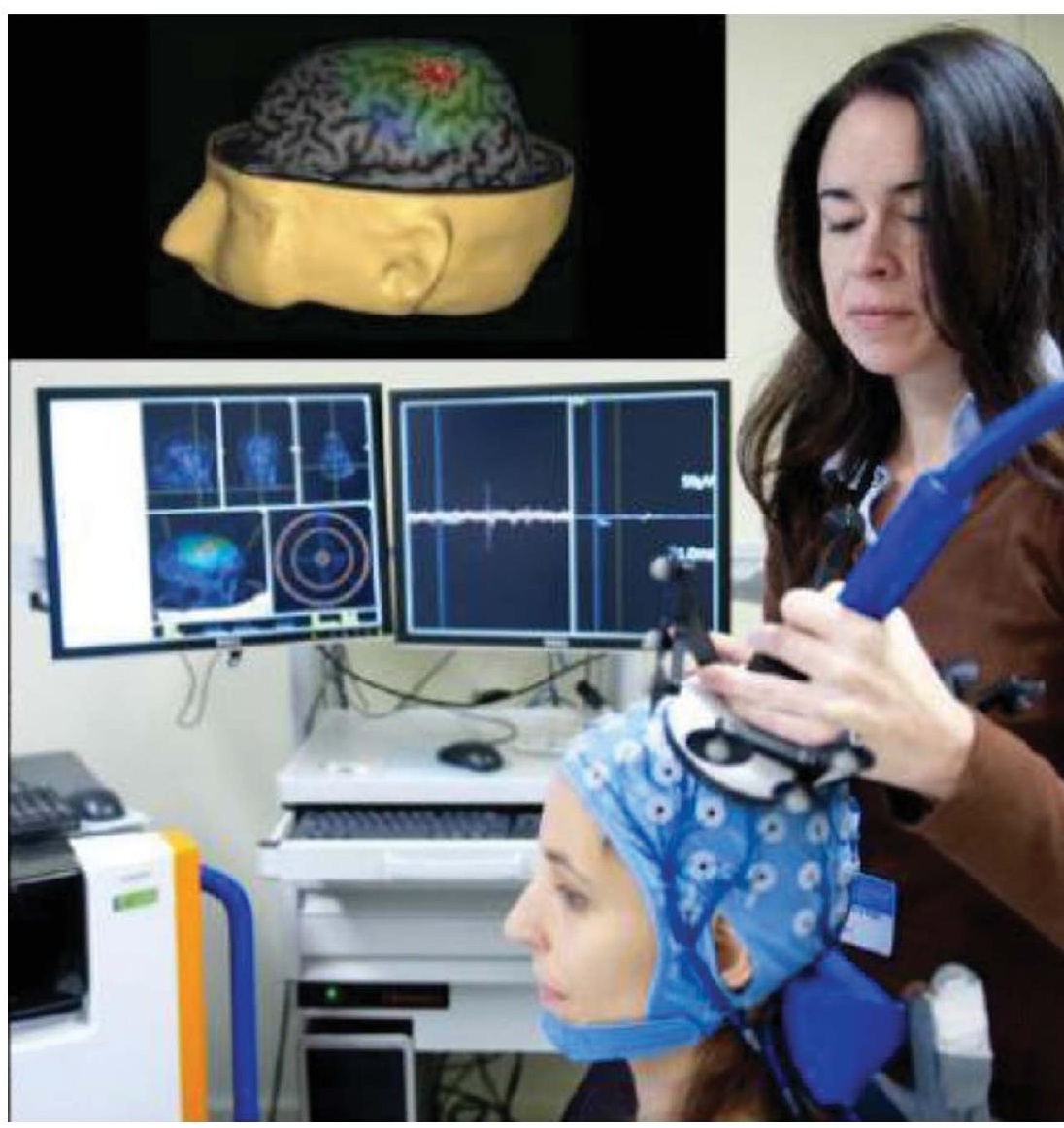

Berenson-Allen Center for Noninvasive Brain Stimulation and the Division of Cognitive Neurology, Department of Neurology, Beth Israel Deaconess Medical Center, Harvard Medical School, Boston; and the Division of Cognitive and Behavioral Neurology, Department of Neurology, Brigham and Women's Hospital, Harvard Medical School, Boston, MA.

Funding information and disclosures are provided at the end of the article. Full disclosure form information provided by the authors is available with the full text of this article at Neurology.org/cp.

Correspondence to: apleone@bidmc.harvard.edu 
conductor adjacent to the coil. In this way, the magnetic field is used to penetrate scalp and skull, while the electric field generates secondary currents leading to neuronal activation in the brain. ${ }^{1-4}$ TMS is capable of noninvasively stimulating a predetermined portion of the neuraxis and of temporarily modulating neuronal excitability (i.e., enhancing or suppressing this) at that site. ${ }^{3,4}$

The expanding adoption of TMS into neuroscience research is reflected in the striking increase in PubMed publications when searching for transcranial magnetic stimulation: from 67 in 1990 to 1,488 in 2000 to 8,699 in 2012. In addition, there has been a commensurately large increase in the use of TMS both diagnostically and therapeutically. The US Food and Drug Administration (FDA) has cleared 2 TMS devices for psychiatric applications. The NeuroStar TMS Therapy system (Neuronetics, Malvern, PA) and, more recently, the Brainsway Deep TMS system (Jerusalem, Israel) have been cleared by the FDA for the treatment of medication-refractory depression. The growing therapeutic effect of TMS upon psychiatric practice is exemplified by the fact that hundreds of clinics in the United States now use an FDA-approved TMS system to treat depression.

In neurology, the Nexstim eXimia Navigated Brain System TMS device (Nexstim Oy, Helsinki, Finland) has FDA approval for presurgical motor and language mapping. The efficacy of TMS as a diagnostic or therapeutic tool in other neurologic contexts has yet to be confirmed. Nevertheless, a growing body of experimental work suggests that the clinical applicability of TMS in neurology, both as a diagnostic instrument and as a means of inducing changes in neural processing to achieve therapeutic gains, may soon be further expanded. This review will focus on mechanisms of action of TMS. It will then survey data from studies that suggest possible clinical applications of the methodology to neurology, while clearly delineating which of these applications have and have not been FDA approved.

\section{Methods of TMS application}

TMS can be applied as single pulses, as a pair of pulses (paired-pulse TMS) separated by a given

Video

neurology.org/cp interstimulus interval (ISI) and applied to the same or different brain areas, or as repetitive trains of stimulation (rTMS). ${ }^{3,4}$ Different forms of TMS application are schematically depicted in figure e-1. Single-pulse TMS is commonly applied over the primary motor cortex to activate contralateral muscles, a process that can be electrophysiologically recorded as motor evoked potentials (MEPs). These MEPs provide quantifiable attributes such as motor threshold for activation, signal amplitude, and latency, which can be correlated with cortical and corticospinal excitability. ${ }^{3,4}$ Physiologically, depending on the orientation of the TMS coil held over the scalp, TMS is thought to primarily exert its influence on corticospinal excitability through its effects upon synaptic transmission (which are reflected as indirect waves [I waves] in the MEP), or to more closely match the effects of transcranial electrical stimulation (TES) (which directly stimulates axons of descending motor pathways and which are reflected as direct waves [D waves] in the MEP). ${ }^{3,5}$ Paired-pulse TMS further allows for the assessment of more cortical-specific excitability. ${ }^{3}$

Following repetitive TMS (rTMS), neuronal excitability can be altered for a sustained period that outlasts the time of stimulation. Depending on the parameters of rTMS applied, this excitability can be facilitated or suppressed. ${ }^{3,4}$ Moreover, the effects of TMS propagate transsynaptically to networks of regions anatomically and functionally connected to the stimulation site. ${ }^{4,6}$ The neurobiologic susbstrates of the effect of TMS remain insufficiently understood, but human and animal experiments are providing valuable insights. For example, acute effects of TMS appear to involve shifts in the ionic equilibrium around cortical neurons or the storage of charge directly from stimulation. ${ }^{4}$ More lasting effects seem to involve distributed brain network impacts, changes in oscillatory activity, specific neurochemical releases, and use-dependent mechanisms of plasticity, including synaptic modifications. ${ }^{4}$

TMS can sometimes be associated with mild transient headache or mild neck or facial discomfort (often caused by muscle contraction induced by the stimulation). Hearing loss, 


\section{TMS can be applied to cortical regions beyond the motor cortex, where it can evoke local field potentials and regional brain activation, which can be captured by coupling TMS with other imaging modalities.}

transient mood or cognitive changes, and syncope have also been observed but are far less common. The most worrisome consequence of TMS, particularly rTMS, is seizure induction. However, when applied with established safety parameters (e.g., with respect to stimulation intensity and duration), seizures are exceedingly rare. ${ }^{4}$ Careful screening of potential recipients of TMS is also important to lower the likelihood of seizure induction. For example, potential recipients should be screened for factors that might lower their seizure threshold (e.g., medications that may be epileptogenic). ${ }^{4}$ In the case of pregnancy, it is unlikely that TMS to the scalp would affect the fetus given the rapid rate of attenuation of the magnetic field with distance. ${ }^{4}$ However, pregnant women should only receive TMS on a case-by-case basis after a risk-benefit ratio has been assessed, and spinal stimulation should be avoided entirely. ${ }^{4}$ Thus, when used within established guidelines, in appropriate patients and in the appropriate setting (e.g., with trained personnel), TMS is considered to be safe.

\section{Established applications of TMS}

Presurgical motor mapping As stated above, the US FDA has approved TMS for presurgical motor and language mapping. By taking advantage of stereotactically guided neuronavigation, TMS can target cortical regions defined by the individual's own brain MRI and thereby systematically probe motor responses, as measured by MEPs induced in different target muscles. As a result, it becomes possible to assess what areas of cortex can be safely excised during tumor resection, or during the removal of an epileptogenic focus, without inducing significant postoperative motor deficits. ${ }^{7-9}$ TMS is able to perform these functions with accuracy profiles similar to those observed with invasive cortical mapping approaches. ${ }^{?}$

Presurgical language mapping The utility of TMS as a means to probe language function dates back to the original demonstration of its ability to induce speech arrest. ${ }^{10}$ More recent investigations have directly compared rTMS to intracarotid amobarbital test (Wada test) in determining hemispheric language dominance, with varying results. ${ }^{11}$ A recent study assessed language function in 20 neurosurgical patients with left hemispheric lesions via an object naming task. The authors found good concordance between presurgical language mapping with TMS and intraoperative language mapping with direct cortical recording in awake patients. ${ }^{12}$

Treatment-refractory major depression Repetitively administered TMS, when delivered to the dorsolateral prefrontal cortex (DLPFC), is an established treatment for certain forms of medication-refractory major depressive disorder (MDD). In these cases, rTMS is typically delivered as high-frequency stimulation to left DLPFC or, less commonly, as low-frequency stimulation to right DLPFC over daily sessions. The efficacy of TMS in treating depression has been supported by large randomized, sham-controlled trials and several meta-analyses. ${ }^{13}$ While the parameters, location, and duration of stimulation in the treatment of depression have yet to be standardized, TMS systems commonly deliver $10-\mathrm{Hz}$ stimulation in daily sessions, 5 sessions per week over a period of 2-6 weeks. One of the 2 FDA-approved TMS systems utilizes a uniquely configured stimulation coil (H-coil), which enables deeper stimulation of the human brain. Duration of stimulation, efficacy, and side-effect profile appears 
Figure 1 Neuronavigation equipment and a potential clinical application of transcranial magnetic stimulation

A TMS (i)
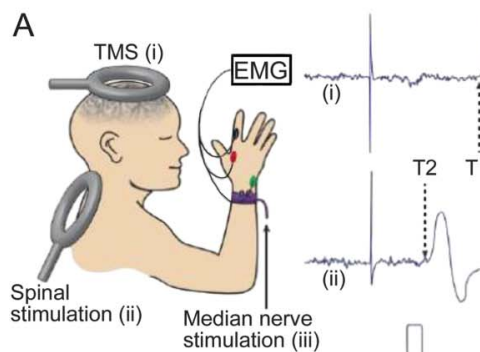

$\int_{1} 1 \mathrm{mv}$
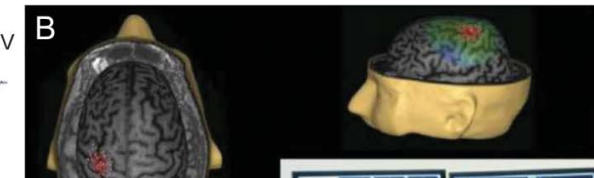

(ii) $\iint_{0} \mid 1 \mathrm{mV}$

T2 T1
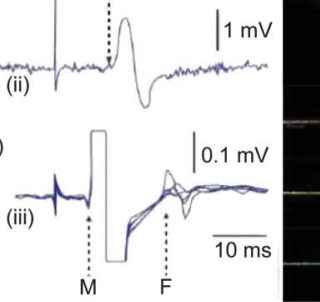

)

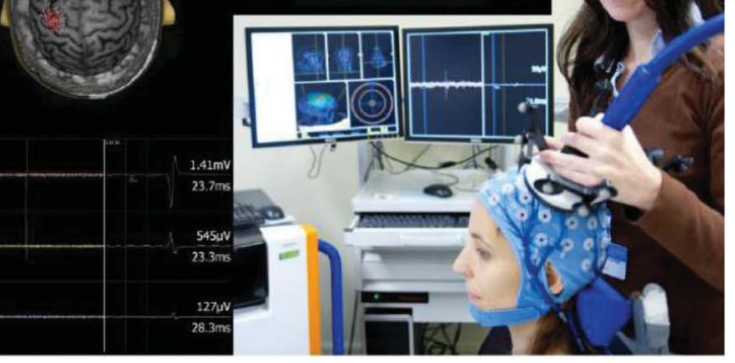

(A) A schematic representation of how central motor conduction time (CMCT) is calculated. Motor evoked potentials (MEPs) are shown following cortical stimulation (i) and after cervical spinal root stimulation (ii). Note that the EMG baseline during cortical stimulation is active because $\mathrm{CMCT}$ is typically measured with the target muscle under slight voluntary contraction in order to reduce the MEP latency to the target muscle. CMCT is calculated by subtracting the onset latency of the MEP resulting from spinal stimulation (T2) from the onset latency of the MEP induced by the cortical stimulation (T1). An alternative method is to measure the F-wave following median nerve stimulation (iii) in order to assess peripheral conduction time. This can allow more precise measurement of the CMCT. $F=$ onset latency of F-wave by median nerve stimulation; $M$ = onset latency of $M$-wave by median nerve stimulation. (B) The Nexstim eXimia Navigated Brain System (NBS) TMS device makes use of stereotactic neuronavigation to target a cortical region that is predetermined on the basis of the subject's own individualized MRI. In this example, the NBS system is being employed to assess the subject's resting motor threshold based on stimulation of the subject's left primary motor cortex hand area (M1). Each red dot on the cortical surface (right panel) represents a single stimulation point in an attempt to locate the hotspot in M1 that will yield the most robust MEP in the contralateral first dorsal interosseous muscle (depicted in the left panel). Once located, this hotspot is stimulated at varying intensities to determine the subject's motor threshold. In this example, the determined motor threshold will be used to perform concurrent TMS and EEG.

to be similar to that of the more conventional system, though comparative studies or data are not yet available.

\section{Research suggesting potential diagnostic applications of TMS in neurology}

TMS can measure corticospinal integrity through the ascertainment of central motor conduction time (CMCT), an index of the time between motor cortex stimulation and the firing of spinal motor neurons (figure 1A). Physiologically, CMCT is typically assessed by subtracting the conduction time between anterior horn cell motor neurons and muscle (i.e., the peripheral conduction time) from the cortical to muscle conduction time. ${ }^{3}$ Mechanistically, peripheral conduction time can be assessed in 1 of 2 ways: either by directly stimulating over the spine to activate spinal roots, or by making use of the $\mathrm{F}$ wave (the muscle response elicited by antidromically stimulating the anterior horn cell following peripheral nerve stimulation). The formula for calculating peripheral conduction time is then determined as ( $\mathrm{F}$ wave latency $+\mathrm{M}$ wave latency -1$) / 2$, where the $\mathrm{M}$ wave is that elicited from directly stimulating the peripheral nerve. Of the 2 methods for assessing peripheral conduction time, direct spinal stimulation is considered less accurate because of the large degree of variability in the extent to which spinal roots are stimulated (e.g., owing to anatomical variability). ${ }^{14}$ Clinically, CMCT could potentially be used to assess the severity of myelopathy in cord compression, the functional level of a cord compression, as a tool to differentiate myelopathy from motor neuron disease, or possibly as a means of predicting functional recovery in MS. ${ }^{3}$ Further, because the procedure for estimating CMCT involves single-pulse TMS, it would not be associated with substantial safety concerns. ${ }^{4}$

TMS can be applied to cortical regions beyond the motor cortex, where it can evoke local field potentials and regional brain activation, which can be captured by coupling TMS with 


\section{In the case of localization-related epilepsy, TMS can be used to directly target a documented cortical seizure focus, or to stimulate an accessible cortical target connected to a subcortical focus.}

other imaging modalities, such as EEG and functional MRI (fMRI). ${ }^{6,15}$ Furthermore, TMS to nonmotor areas can be used to assess behavioral effects induced by the stimulation. In this manner, the cognitive impacts from stimulating a particular brain region or brain network can be gauged. For example, as stated above, there is FDA clearance to use TMS as a tool to elaborate language pathways. The use of TMS as a tool to map processing in other cognitive domains, however, remains relegated to research at this time.

\section{Research suggesting potential therapeutic applications of TMS in neurology}

While TMS has not been FDA-approved as a treatment modality for neurologic disease, studies are underway to explore the potential therapeutic use of TMS for a diverse array of neurologic disorders. Available evidence of efficacy to date is limited to proof-of-principle studies and often to only acute effects. Larger and better-controlled clinical trials that address the durability of the potential benefits are needed to explore the therapeutic potential of TMS. With respect to the putative durability of any clinical effects, it is anticipated that any efficacious therapeutic application of TMS would require multiple repeated sessions of stimulation, as is the case in the treatment of major depression.

Epilepsy Some forms of rTMS (e.g., low-frequency, 1-Hz rTMS or continuous theta burst TMS) can suppress cortical excitation and induce LTD-like changes (possibly through GABAergic modulation). ${ }^{16}$ Therefore, these have been investigated as an abortive strategy for seizures and can be useful, for example, in focal status epilepticus or epilepsia partialis continua. ${ }^{16}$ In the case of localization-related epilepsy, TMS can be used to directly target a documented cortical seizure focus, or to stimulate an accessible cortical target connected to a subcortical focus. However, the antiepileptic efficacy of TMS is closely tied to the relative success of targeting the epileptogenic regions. As such, medial temporal lobe foci are not amenable to TMS treatment with current methods. Reductions in seizure frequency following rTMS have been demonstrated in many but not all randomized sham-controlled trials. ${ }^{16}$ There is currently no FDA approval for the use of TMS in the treatment of epilepsy.

Stroke rebabilitation Following a stroke, there appears to be a hemispheric imbalance with excessive inhibition from the healthy to the lesioned hemisphere. TMS has been employed either to suppress contralesional cortex (with low-frequency stimulation) or to augment cortical excitability in ipsilateral, perilesional cortex (with high-frequency stimulation). ${ }^{3,8}$ In addition to enhancing motor recovery after stroke, other preliminary studies have shown promising results using similar TMS paradigms to improve aphasia and hemispatial neglect following stroke. ${ }^{17}$ Nevertheless, in this context, TMS or other noninvasive techniques such as transcranial direct current stimulation may be most effective when combined with other rehabilitation strategies. Issues surrounding patient characteristics, timing of the TMS intervention, and effective stimulation parameters will need to be determined for both FDA approval and widespread clinical use to treat deficits following stroke.

Movement disorders There are mounting data from sham-controlled studies to support the use of high-frequency rTMS to premotor and primary motor cortices in improving 
bradykinesia and freezing in Parkinson disease (PD)., ${ }^{3,7}$ These effects might be mediated by transsynaptic modulations of the nigrostriatal-thalamocortical circuit. ${ }^{8,18}$

Low-frequency rTMS aiming to suppress cortical excitability has been applied to similar motor and premotor regions as a way of decreasing L-Dopa-induced dyskinesias with some success, and cerebellar targets are also being explored. ${ }^{18}$ TMS has also been investigated as a treatment option in dystonias, with controlled studies demonstrating therapeutic benefits from targeting dominant primary motor cortex (M1) in focal hand dystonia. ${ }^{3}$ Multicenter trials are currently underway to better define the effectiveness of TMS for motor symptoms in $\mathrm{PD}$, as a first step toward possible FDA approval.

Chronic pain TMS has shown promise in decreasing chronic pain in patients with neuropathic pain (e.g., that resulting from peripheral nerve, spinal cord, or central sensory pathway injury), fibromyalgia, and complex regional pain syndrome. ${ }^{3,8}$ The applicability of TMS to visceral pain (e.g., chronic pancreatitis) has also been reported. ${ }^{8}$ Stimulation sites for these studies commonly involve the primary somatomotor cortex (SM1), with the supposition that pain suppression occurs by way of the activation of interconnected thalamic nuclei, and through downstream effects upon brainstem and spinal cord nuclei and interconnected limbic regions. ${ }^{8}$ Ongoing studies are aimed at better defining the target of stimulation, the parameters for stimulation, and the patient characteristics that predict response. However, effect sizes in patients who do respond to TMS can be substantial, and duration of benefit clinically significant.

Tinnitus Application of rTMS over primary auditory cortex appears to decrease tinnitus, and repeated sessions of rTMS to this region have been shown to induce more persistent suppression of tinnitus in several controlled studies. ${ }^{19}$ Most studies have applied low-frequency rTMS, but the optimal parameters are unclear. Furthermore, the effects of rTMS show high degrees of interindividual variability (possibly related to variability in age, hearing loss, and the chronicity of the tinnitus). ${ }^{16}$

\section{DISCUSSION}

In contrast to other neurostimulation approaches, TMS is noninvasive. Similar to these approaches, TMS is capable of causally assessing brain-behavior relationships, which has clear diagnostic implications for neurologic disorders. Such diagnostic potential has already been realized in the now approved use of TMS for presurgical motor and language mapping. While there are currently no FDA-approved treatment regimens for neurologic disorders, mounting experimental evidence suggests that TMS might offer therapeutic promise for neurologic conditions wherein current treatment options are substantially suboptimal or lacking altogether.

\section{REFERENCES}

1. Cohen D, Cuffin BN. Developing a more focal magnetic stimulator: part I: some basic principles. J Clin Neurophysiol 1991;8:102-111.

2. Roth BJ, Cohen LG, Hallett M, et al. A theoretical calculation of the electric field induced by magnetic stimulation of a peripheral nerve. Muscle Nerve 1990;13:734-741.

3. Hallett M. Transcranial magnetic stimulation: a primer. Neuron 2007:55:187-199.

4. Rossi S, Hallett M, Rossini PM, et al. Safety, ethical considerations, and application guidelines for the use of transcranial magnetic stimulation in clinical practice and research. Clin Neurophysiol 2009; 120:2008-2039.

5. Amassian VE, Stewart M, Quirk GJ, et al. Physiological basis of motor effects of a transient stimulus to cerebral cortex. Neurosurgery 1987:20:74-93.

6. Hampson M, Hoffman RE. Transcranial magnetic stimulation and connectivity mapping: tools for studying the neural bases of brain disorders. Front Syst Neurosci 2010;4:1-8.

7. Krieg SM, Shiban E, Buchmann N, et al. Utility of presurgical navigated transcranial magnetic brain stimulation for the resection of tumors in eloquent motor areas. J Neurosurg 2012;116:994-1001.

8. Najib U, Bashir S, Edwards D, et al. Transcranial brain stimulation: clinical applications and future directions. Neurosurg Clin N Am 2011;22:233-251. 
9. Picht T, Schmidt $S$, Brandt $S$, et al. Preoperative functional mapping for rolandic brain tumor surgery: comparison of navigated transcranial magnetic stimulation to direct cortical stimulation. Neurosurgery 2011;69:581-588.

10. Pascual-Leone A, Gates JR, Dhuna A. Induction of speech arrest and counting errors with rapid-rate transcranial magnetic stimulation. Neurology 1991;41:697-702.

11. Devlin JT, Watkins KE. Stimulating language: insights from TMS. Brain 2007;130:610-622.

12. Picht T, Krieg SM, Sollmann N, et al. A Comparison of language mapping by preoperative navigated transcranial magnetic stimulation and direct cortical stimulation during awake surgery. Neurosurgery 2013;72:808-819.

13. Slotema CW, Blom JD, Hoek HW, et al. Should we expand the toolbox of psychiatric treatment methods to include Repetitive Transcranial Magnetic Stimulation (rTMS)? A meta-analysis of the efficacy of rTMS in psychiatric disorders. J Clin Psychiatry 2010;71:873-884.

14. Barker AT, Freeston IL, Jalinous R, et al. Magnetic stimulation of the human brain and peripheral nervous system: an introduction and the results of an initial clinical evaluation. Neurosurgery 1987; 20:100-109.

15. Levy WJ, Amassian VE, Schmid UD, et al. Mapping of motor cortex gyral sites non-invasively by transcranial magnetic stimulation in normal subjects and patients. Electroencephalogr Clin Neurophysiol Suppl 1991;43:51-75.

16. Rotenberg A. Prospects for clinical applications of transcranial magnetic stimulation and real-time EEG in epilepsy. Brain Topogr 2010;22:257-266.

17. Miniussi C, Cappa SF, Cohen LG, et al. Efficacy of repetitive transcranial magnetic stimulation/transcranial direct current stimulation in cognitive neurorehabilitation. Brain Stimul 2008;1:326-336.

18. Koch G, Brusa L, Carrillo F, et al. Cerebellar magnetic stimulation decreases levodopa-induced dyskinesias in Parkinson disease. Neurology 2009;73:113-119.

19. Langguth B, de Ridder D, Dornhoffer JL, et al. Controversy: does repetitive transcranial magnetic stimulation/transcranial direct current stimulation show efficacy in treating tinnitus patients? Brain Stimul 2008;1:192-205.

\section{STUDY FUNDING}

M. Eldaief was supported by NIH grant 5K23MH099413.

\section{DISCLOSURES}

M. Eldaief reports no disclosures. D. Press has received travel and speaker honoraria from Headache Cooperative of Northeast and the Medical School of Singapore; receives publishing royalties from $U_{p}$ ToDate; receives approximately $25 \%$ of his salary from his role as clinical director for the Center for Noninvasive Brain Stimulation; and receives research support from Fidelity Bioscience Research Initiative and the NIH. A. Pascual-Leone serves on Scientific Advisory Boards for Nexstim, Neosync, Starlab, Neuronix, Allied Minds, and Novavision; serves as Associate Editor for European Journal of Neuroscience and Frontiers in Neuroscience; is listed as coinventor in several patents related to real-time integration of TMS with EEG and functional imaging; and receives research support from Nexstim, Neuronix, the NIH (NEI, National Institute of Neurological Disorders and Stroke, NCRR), Michael J. Fox Foundation, Berenson-Allen Foundation, and RJG Family Foundation. Full disclosure form information provided by the authors is available with the full text of this article at Neurology.org/cp. 


\section{Related articles from other AAN physician and patient resources}

Neurology ${ }^{\circledR} \bullet$ www.neurology.org

Theta-burst stimulation of the left hemisphere accelerates recovery of hemispatial neglect January 3,2012;78:24-20.

Intermittent theta-burst transcranial magnetic stimulation for treatment of Parkinson disease February 15,2011;76:601-609.

\section{Continuum ${ }^{\circledR} \quad$ @ www.ContinuumJournal.com}

Introduction: The evolving field of neurorehabilitation June 2011;443-448.

Emerging therapies in neurorehabilitation June 2011;530-544.

\section{Neurology Now ${ }^{\circledR} \bullet \quad$ www.neurologynow.com}

Sounds of silence

February/March 2012;8:20-25.

This way in: ADHD causes motor skill problems June/July 2011;7:13-14.

\section{Neurology Today ${ }^{\circledR}$}

- www.neurotodayonline.com

In older adults, less frontal volume and slow-wave activity add up to worse memory March 21,2013;13:5-6.

Findings of impaired motor cortex inhibition in ADHD offers potential for quantitative evaluation of children February 17,2011;11:31-32. 